\title{
5
}

\section{BPR for Developing Information Systems - a case study}

\author{
Elayne Coakes and Jim Coakes
}

School of Business Systems, Faculty of Business, University of Luton, Luton, Beds, LU1 3JU

School of Business, Thames Valley University, Slough, Berks, SL1 1YG

\begin{abstract}
The use of BPR techniques in CIS development is useful in the strategy, analysis and requirements definition phases, but only if it is part of a business wide quality improvement initiative. Its major strengths lie in techniques for balanced empowerment of stakeholders and levelling of power from an hierarchical base point. In addition, using BPR techniques in CIS development may impact positively on the quality of the CIS.

This paper reports a detailed case study in the use of BPR techniques in CIS development. The system was developed during 1992 and implemented at the end of 1993. In 1994, as this paper is being written, the system is being used successfully and stakeholders are looking for further enhancements to extend its area of operation. It is clear from this that expectation success has been achieved and that in terms of software quality the system fulfils many of McCall's criteria.
\end{abstract}

Keyword Codes: D.2.1,H.4.1

Keywords: Software Engineering, Requirements, Information Systems Applications, Office Automation.

\section{INTRODUCTION}

The Company under review is an international engineering, construction and project management contractor, which is currently shifting from individual attainment to group quality. To achieve this they had recently introduced a comprehensive policy of continuous improvement through BPR.

\section{COMPUTER INFORMATION SYSTEM (CIS) DEVELOPMENT}

In Hirschheim and Klein [1989 ${ }^{1}$ four paradigms of Information Systems development are discussed:

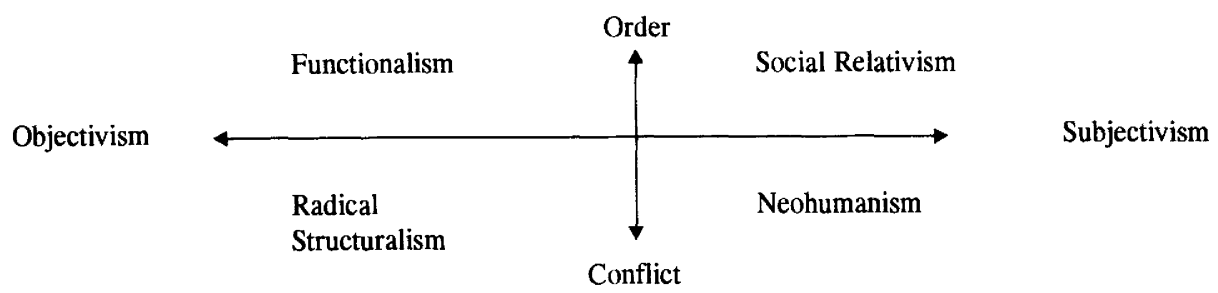


They identify the story of the Analyst as Systems Expert (the orthodox approach to systems development) as lying within the functionalism paradigm. The assumption to be made is that specification should be as objective as possible. The resolution of issues is not within the domain of the systems developer, he is primarily the expert. Business Process Re-engineering, we would argue, falls into the story of the Analyst as Facilitator within the Social Relativist paradigm. The system developer should work from within the users' perspectives and help them find their preferred views, his role being to find out what system makes sense. Thus any system that meets with the approval of the affected parties is legitimate. The facilitator helps stimulate reflection and co-operation, using appropriate tools and methods, and favours strong participation.

The assumption behind this story corresponds to Lyytinen and Hirschheim's view [1987] ${ }^{2}$ that expectation failure (where the CIS does not fulfil stakeholder's expectations) is related to the values and perceptions of information system stakeholders.

\subsection{Developing Better Systems}

Two major areas are identified as producing systems that are more successful, 'conflict resolution' and (improving) 'requirements definition'. A recent Price Waterhouse ${ }^{3}$ survey found that of all respondents $45 \%$ mentioned poor requirements definition, $34.5 \%$ little user involvement and $29.5 \%$ political issues, as reasons for CIS failures.

\subsubsection{Conflict Resolution}

Mumford [1983] $]^{4}$ says:

'all change involves some conflicts of interest. To be resolved, these conflicts need to be recognised, brought out into the open, negotiated and a solution arrived at which largely meets the interest of all parties in the situation... successful change strategies require institutional mechanisms which enable all these interests to be represented, and participation provides these.'

Activities are ultimately controlled by those who perform them. People who do not have a say in the decisions on how these activities are to be changed, may not abide by those decisions. Jones and Kydd [1988] $]^{5}$ would go on to say that equivocality (ambiguity or the absence of clarity) resolution is critical to realising improved productivity. The use of group meetings, integrators and face to face contact help to resolve equivocality.

\subsubsection{Requirements Definition}

The Company traditionally used Information Engineering (IE) as the preferred development methodology. IE falls into the functionalism paradigm. Under IE the specification is designed to be as objective as possible and it lays great emphasis on data analysis as data is more stable than processes. Diagrams are used for communication and users are encouraged to participate in system design. The difficulty is that users may not understand completely the terminology and technical language used. Fitzgerald $[1990]^{6}$ argues that the traditional approach to software documentation produces documents that are poorly understood by users. Kit Grindley [1991] ${ }^{7}$ quotes a manager as saying: 'How would you describe a journey into the unknown, a place where your compass doesn't work, the sun and the stars are replaced by elusive rainbows... The language is strange, the customs new, the pathways intricate and mysterious and you depend on local guides and native bearers.'

Mumford $[1985]^{8}$ says that any definition of system requirements should concentrate first on business problems and needs. She advocates the use of a design group consisting of users and their representatives as well as technical specialists to debate system process and design.

\subsection{BPR in CIS Development}

A corporate culture embodies the shared values, meanings and understanding about what is important for the organisation. Job structure follows naturally from culture and so Kydd and Jones 
$(1989)^{9}$ assert one of the reasons so many IS development methodologies fail is the difficulty in changing job structure without first changing culture. Yet most IS implementations change job functions and structure axiomatically. BPR expressly sets out to change culture and mind-set.

\subsubsection{A Measure of Success - Improved Software Quality.}

How do we assess software quality? Pressman $[1992]^{10}$ defines software quality as:

'conformance to explicitly stated functional and performance requirements, explicitly documented development standards, and implicit characteristics that are expected of all professionally developed software.... Software requirements are the foundation from which quality is measured. Lack of conformance to requirements is lack of quality.'

McCall et al [1977] $]^{11}$ identify ten quality factors: maintainability; flexibility; testability; portability; re-usability; interoperability; correctness; reliability; efficiency; integrity and usability. But many of these quality measures and metrics are inappropriate in looking at BPR at work. BPR and continuous improvement techniques measure quality by process improvement and therefore CIS development designed using these techniques need to use this type of measurement also. We will show in the case study that process improvement and the use of BPR techniques help resolve conflicts, improve requirements definition and produce quality software.

\section{CASE STUDY}

The Company needs to account carefully for national and international telephone charges. Substantial charges are incurred working on projects across the Eastern Hemisphere and Europe, and a charge from any phone needed to be allocated to a staff member, a department or a client project.

\subsection{Initial Situation - How Did The Process Become So Complex?}

A CIS for recharging telephone costs had been developed by outside consultants in 1984, and transferred to a new platform in 1990 . The CIS became more complex as particular features and requirements has been built in. The system used batch processes to handle 2 weeks' phone calls at a time to produce journal vouchers and other input for the financial billing systems. These processes also identified incorrect data well after it had entered the system. When the old CIS had problems, and outputs had to be produced under time pressure, sometimes an extra data correcting batch process would be added in to make the faulty output usable by a financial system. Sometimes this extra process became a regular feature of future runs of the system.

\subsection{Systems Analysis And Development - How Did BPR Techniques Help?}

The Company had recently introduced BPR, training senior management in the approaches and outcomes at residential courses run by external consultants. However, at this point the supervisors and other staff had only received a brief introduction to BPR. Initial staff reaction to BPR was 'Oh no not another cure all management technique, it will be like all the others, they come in every few years then fade away'. This highlights a degree of cognitive dissonance between staff and management.

The MIS manager responsible for running the inefficient legacy system mentioned above, was keen on the benefits of BPR, so he set up a project to examine the processes associated with the telephone charging system. Senior IT and quality management were kept fully informed of the proposed project and its progress.

The MIS Manager set up a team to examine the processes, bringing in the following stakeholders:

$\tilde{\mathrm{n}}$ Staff 'on the ground': Phone operator(s), IT staff responsible for laborious 2 weekly batch work and accounts clerks who received data for further processing.

ñ Management: IT Department, Accounting and Office Services. 
Meetings were set up to review the project. As BPR was very new to the Company at the time, a trained facilitator was added to the team to help maintain a 'BPR direction' and assist with implementing the new techniques. There was initial nervousness about this new way of solving problems. This was particularly evident where equal rank was maintained in the meeting room, in an otherwise very hierarchical organisation. At the early meetings it took several hours to agree the basic direction, aims and mission, but this served to maintain 'goal congruence' later among team members. Team members were not given time allowances for attending, but it was assumed that a successful outcome would reward team members with a saving of future efforts. When work pressure was heavy it was therefore hard to keep busy people in the team.

After agreement of aims, the team moved on to assessment of processes, starting off by using Brainstorming to develop ideas of the steps involved in the process. The team identified the departments and groups involved in each process. There was then enough information to draw up Deployment flow charts (DFCs) of the processes. DFCs show process flows, highlighting stakeholder responsibility at each stage. These graphic and comprehensive charts provided a powerful level of insight and understanding of the whole process to an extent which surprised all members. To clarify the (rather complex) DFCs, 'Affinity diagrams' ${ }^{\text {i }}$ were used, grouping together related processes. In order to make decisions, light voting, where participants put a number of points against the various options, was used. This deflated divisive and rigid positions on decision options. When a conflict arose in a meeting, the aim was to resolve it by discussion and sharing of information. Clearly for this information sharing to work fully, it was important that all participants attended meetings regularly.

\subsection{Software Quality - Does BPR Improve It?}

The work performed on this project earned an award from the Company for Continuous Improvement. In particular, the Company was impressed by the team's self starting approach and initiative in improving processes, in line with the principles of BPR. The main users stated that the system fulfilled their key objective of providing correct data, on time, to the finance department. In particular, it now takes two to three days to process a two week period's phone calls, instead of seven to ten days, with one period's work sometimes causing chaos by running into the next period's work. Switchboard staff estimate that they now put half as many hours into processing the data, declining from 25 to 15 hours per week, although the process is still far from fully automated, only relieving them of two work processes. The system's screens and menus were also felt to be very usable.

The system is now reliable, and has not required IT Department intervention at all during the last two working periods (May 1994). The previous level of IT staff involvement was around 25 person hours per week. Users do, however, have a very long list of required enhancements, but there are no IT resources to satisfy these needs. Should staff become available, the system would be relatively easy to amend. Such a large enhancement list (rather than a bug fix list) can be seen as indicating a desire to expand the system's area of involvement. Note that documentation is significantly incomplete and yet the system is operating successfully.

\subsection{BPR vs Other Methodologies}

The Company's usual methodology for system development is Information Engineering. BPR was used to supplement the strategy and analysis stages of the methodology. Essentially, BPR reemphasised the importance of involving all stakeholders in the development throughout the essential early stages. BPR diagrams such as DFCs are directly comparable with Data Flow Diagrams as used

\footnotetext{
' Based on the KJ Method developed by Jiro Kawakita in the 1960's and as taught to the company by Technology Exchange Center (TEC) Inc., Santa Ana, California, 92701
} 
by most system development methodologies. But with BPR, the way the analysis is performed is of more importance than the actual data and the processes.

\section{LESSONS LEARNED}

Lessons learnt during the process included:

$\tilde{\mathbf{n}} \quad$ No process is sacred or fixed

$\tilde{\mathrm{n}} \quad$ All relevant stakeholders must be brought into the development process and involved fully at the requirement definition phase.

$\tilde{\mathbf{n}} \quad$ Discussions are most effective when staff rank is ignored.

$\tilde{\text { n }} \quad$ BPR techniques help empower individuals and break down departmental barriers.

$\tilde{\mathbf{n}} \quad$ Commitment of the whole development team, of both IT and non IT staff to shared goals, will contribute significantly to the success of the system.

\section{CONCLUSION}

The use of BPR techniques in CIS development is useful in the strategy, analysis and requirements definition phases, but only if it is part of a business wide quality improvement initiative. Its major strengths lie in techniques for balanced empowerment of stakeholders and levelling of power from an hierarchical base point.

The case study system was developed during 1992 and implemented at the end of 1993. In 1994, as this paper is being written, the system is being used successfully and stakeholders are looking for further enhancements to extend its area of operation. It is clear from this that expectation success has been achieved and that in terms of software quality the system fulfils many of McCall's criteria.

\section{REFERENCES}

1. Hirschheim, R. and Klein, H.K.: 'Four Paradigms of Information Systems Development', Communications of the ACM, 32 10, October 1989, pp199-1216.

2. Lyytinen, K. and Hirschheim, R.: Information Systems Failures - a survey and classification of the empirical literature', Oxford Surveys in Information Technology, 4, 1987, pp257-309.

3. Price Waterhouse Survey of Computer Failures, 1989.

4. Mumford, E.: 'Designing Human Systems', Manchester Business School., 1983.

5. Jones, L.H. and Kydd,C.T.: 'An Information Processing Framework for Understanding Success and Failure of MIS Development Methodologies', Information and Management, 15, 1988, pp263-71.

6. Fitzgerald, G.: 'Achieving Flexible Information Systems: the case for improved analysis', Journal of Information Technology, 5 , 1990, pp5-11.

7. Grindley, K.: 'Managing IT at Board Level: the hidden agenda exposed', Pitman, 1991, pp149.

8. Mumford, E.: 'Defining Systems Requirements to Meet Business Needs: a case study example', The Computer Journal, 282 , 1985, pp97-104.

9. Kydd, C.T. and Jones, L.H.: 'Corporate Productivity and Shared Information Technology', Information Management 17, 1989, p277-282.

10. Pressman, R.: 'Software Engineering: A practitioner's approach', McGraw-Hill. 1992, pp150.

11. Mccall, J., Richards, P., Walters, G..Factors in Software Quality', NTIS AD-A049-014, 015, 055. November 1977. 